\title{
A Proposed Framework for Combining Smart Environment and Heuristic Diagnostic Teaching Principles in Order to Assess Students' Abilities in Math and Supporting them during Learning
}

\author{
Ardiana Sula* \\ Evjola Spaho† \\ Leonard Barolli§ \\ Rozeta Miho* \\ *Polytechnic University of Tirana, Faculty of Information Technology \\ Mother Teresa Square, Nr. 4, Tirana, Albania \\ E-mail: ardianasula@gmail.com, rmiho@fti.edu.al \\ ${ }^{\dagger}$ Graduate School of Engineering, Fukuoka Institute of Technology (FIT) \\ 3-30-1 Wajiro-Higashi, Higashi-Ku, Fukuoka 811-0295, Japan \\ E-mail: evjolaspaho@hotmail.com

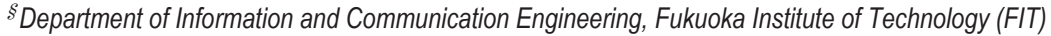 \\ 3-30-1 Wajiro-Higashi, Higashi-Ku, Fukuoka 811-0295, Japan \\ E-mail: barolli@fit.ac.jp
}

Doi:10.5901/mjss.2014.v5n2p187

\section{Abstract}

In this paper, we propose the use of the smart environment based on Internet of Things (IOT), P2P technology and Heuristic Diagnostic Teaching principles for identifying learning abilities in mathematics and creative traits for students that are diagnosed within Autism Spectrum disorder (ASD). Many children with autism are highly interested and motivated by smart devices such as computers and touch screen tablets. These types of assistive technology devices get children with autism to interact, make choices, respond, gain new communication skills and create P2P communication between children, caregivers and therapists. Our proposed system uses JXTA-Overlay platform and SmartBox device to support students during their learning process by getting and maintaining their concentration on a given task. We propose to evaluate children's ability in math using the Heuristic Diagnostic Teaching principles. Our new system combined with various visual systems, such as objects, photographs, pictures, realistic drawings, line drawings, and written words, can be used with assorted modes of technology, as long as the child can readily comprehend the visual representation. Vocabulary skills, mathematics skills and other life skills can be taught through our proposed system. We propose to use different assessment tools to learn about students' ability in math.

Keywords: P2P technology, Internet of Things, Smart environment, SmartBox, JXTA-Overlay, RFID, Assistive technologies, Heuristic Diagnostic Teaching.

\section{Introduction}

Most of the socially assistive research to date is focused on Autism Spectrum Disorder (ASD). Autism is a neurological disorder that affects the ability to communicate and interact socially. One way to cope with this problem is using assistive technologies and finding ways how to benefit from the use of technology to help these children. Many individuals with cognitive disabilities, developmental and social disorders constitute another growing population that may benefit from assistive applications in the contexts of special education, therapy, and training. The cause of the increasing number of children with autism is not yet known. However, early intervention is critical to enabling a positive long-term outcome, and even with early intervention, many individuals will need high levels of support and care throughout their lives.

An assistive smart environment has the potential to enhance the quality of life for broad populations of users: elderly, individuals with physical impairments and those in rehabilitation therapy, and individuals with cognitive disabilities and developmental and social disorders [1]. 
The advance of the technology is leading to smart objects being capable of identifying, locating, sensing and connecting and thus leading to new forms of communication between people and things; and, things themselves. Nowadays, the researchers and developers are focused on developing various smart devices that allow users to control and monitor events in consumer-based appliances, home electronics, and home security systems. As these devices become more common, the need will increase for a home networking strategy that will allow all data, voice, and smart devices to be accessed at anytime from anywhere.

Internet of Things (IoT) will be a key part of the future Internet, and Radio Frequency Identification (RFID) tags, allow objects to be uniquely identified, to determine the location, to sense changes in physical data and to connect and communicate with a corresponding transponder. Children with autism are the subjects of the research presented here, because there is no current cure for autism and the primary care goal is in creating a supportive environment.

In this paper, we propose a system based on an assistive smart environment for identifying students' learning abilities in math and their creativity traits using the Heuristic Diagnostic Teaching (HDT) process. Our proposed system is going to use the computer, sensors, RFID tag reader and SmarBox device. Many specialists and researchers support the use of ICT to support learning process for students with ASD. There are many programs available for teaching children with special needs, and the benefit of using ICT is that it allows for a multi-sensory approach such as visual, sound, interactive aspect [2].

Many children with autistic spectrum condition show good ability in the number area of mathematics, but in order for this group of children to develop a real understanding of mathematics and the function of it in their everyday life it is required to develop more creative methods to succeed in their learning. Many of autistic children are visual learners and the use of ICT solutions that combine multi-sensory approach can be very helpful.

Children with a diagnosis of autism vary greatly in their strengths/weaknesses, likes/dislikes and general personality and approach to life and therefore cannot be recommended a single teaching method that can be used for all of them. In today's market, there are several teaching materials and methods that can be used based on students' needs. It is very important when designing materials and activities for ASD children, to use "trial and error" to find out what approach is best [3].

Solving word problem might be a difficult task for some students and also for students with ASD. Some of the reasons that the word problems are difficult to be solve are that the students need to understand the problem and find out what is given and what is wanted. It will be easier for them to have the equation ready rather than trying to find out the equation first and find the solution. Knowing for fact that word problems are part of everyday life we can't avoid teaching children how to deal and solve these types of problems.

We are going to provide to children an assistive environment so they can become more actively engaged during the diagnose process and later use this system to support students during their learning. Our proposed system doesn't intend to substitute face-to-face interaction with the teacher or other professionals, but rather provide a diagnose process for students with autism as a form of active engagement. Our proposed system will provide an environment which is predictable, concrete, self-paced, and promotes better engagement process. The actively engagement of students with ASD in this learning environment can increase attention and decrease anxiety related behaviors [4] .

The rest of the paper is organized as follows. In Section 2, we give an overview of the related work. In Section 3 , we present the proposed system to support students with autism during their learning. In Section 4, we give a proposal of using combination of smart environment and HDT principle. In Section 5, we present a case study to evaluate students in Math subject. Finally, in Section 6, we present conclusions and future work.

\section{Related Work}

\subsection{Networking Technologies}

Advances in data collection technology, such as embedded devices and RFID technology have led to an increasing number of smart devices (see Fig. 1) that allow users to control and monitor events in consumer-based appliances, home electronics, and home-security systems.

Figure 1: Smart devices in children environment. 


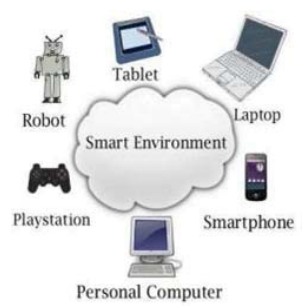

As these devices become more common, the need will increase for a home networking strategy that will allow all data, voice, and smart devices to be accessed at anytime from anywhere [5].

Home networking is the collection of elements that process, manage, transport, and store the information, enabling the connection and integration of multiple computing, control, monitoring, and communication devices in the home.

Robots are being steadily introduced into modern everyday life and are expected to play a key role in the near future. Assistive robotics in general and socially assistive robotics in particular have the potential to enhance the quality of life for broad populations of users: the elderly, individuals with physical impairments and those in rehabilitation therapy, and individuals with cognitive disabilities and developmental and social disorders [6]. This presents the hope that a robot might be used to teach them social skills and other skills incrementally, and assists in the transfer of this knowledge to interactions with humans.

Peer to Peer (P2P) systems appeared as a new paradigm after client-server and became quite popular for filesharing among Internet users. Such systems benefit from high scalability and fault tolerance. Today research is focused on the design, implementation and deployment of full featured P2P networks that integrate end-devices. P2P is a very good approach to an efficient platform for e-learning and robot control.

A Wireless Personal Area Network (WPAN) is a low range wireless network which covers an area of only a few dozen meters. This sort of network is generally used for linking peripheral devices (like printers, cellphones, tablets and home appliances) or a Personal Digital Assistant (PDA) to a computer, or just two nearby computers, without using a hard-wired connection.

Wireless Sensor Networks (WSNs) are increasingly gaining impact in our daily lives. They are finding a wide range of applications in various domains, including healthcare, assisted and enhanced-living scenarios, industrial and production monitoring, control networks, and many other fields.

\subsection{Internet of Things (IoT)}

The term IoT has recently become popular to emphasize the vision of a global infrastructure of networked physical objects. IOT is a new type of Internet application which enables the things/objects in our environment to be active participants with other members of the network, by sharing their information on a global scale using the same Internet Protocol (IP) that connects to the Internet. The descriptive models for Internet of Things are introduced based on two attributes ("being an Internet", "relating to thing's information") and four different features (only for thing's information, coded by UID or EPC, stored in RFID electronic tag, uploaded by non-contact reading with RFID reader) [7].

The IOT creates human-machine or machine-to-machine communications. In this way the things/objects are capable of recognizing events and changes in their surroundings and are acting and reacting autonomously largely without human intervention in an appropriate way.

Figure 2: IoT model.

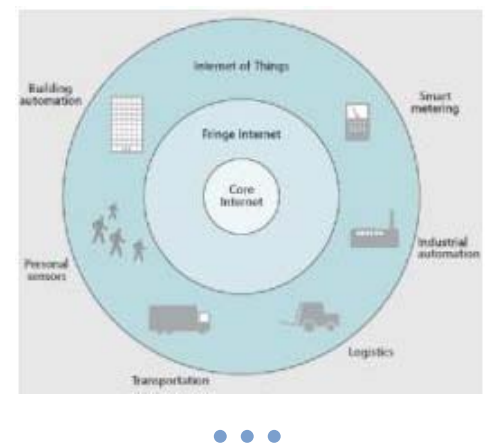


The major objectives for IoT applications and services are the creation of smart environments/spaces and self-aware things for smart transport, products, cities, buildings, energy, health, social interaction and living applications (see Fig. 2). IOT development depends on successful development of RFID technology and design of various new applications and business models. RFID and sensor network technologies will rise to meet this new challenge, in which information and communication systems are embedded in the environment. RFID is primarily used to identify objects from a distance of a few meters, with a stationary reader typically communicating wirelessly and can be used to determine the approximate location of objects provided the position of the reader is known. RFID systems consist of three main components: RFID tags, the RFID readers, and RFID software. The IoT will accelerate and expand, since the IPv6 (Internet Protocol version 6) implementation supports tremendous growth of IP addresses, giving broader ability for every physical thing to connect to the Internet and communicate with any other object.

\section{A New System for Supporting Children with ASD Based on Based on loT and P2P Technologies}

Assistive Technologies (ATs) such as Ambient Assisted Living (AAL) encompasses technical systems to support children with autism in their daily routine to allow an independent and safe lifestyle and an assistive learning environment. The combination of smart technologies results in an applied IoT infrastructure for AAL scenarios. A central AAL paradigm can be realized through the IOT, where the children with autism live in their homes with smart objects, thus smart homes, communicating to the outside world in an intelligent and goal-orientated manner.

We implemented in our previous work the combination of IoT, P2P, Web and sensor technologies for monitoring, checking, controlling the health situation and assisting children with ASD. Our implemented IoT based system is shown in Fig. 3. Our system allows sending information about the children state in real time to therapists using P2P technology and also allows children to interact with other children and parents. Taking advantages of the fact that students with autism are good at using technology, we proposed and implemented a new e-learning system in order to increase the children's concentration by combining computer, projector, SmartBox and sensors.

A study presented in 2008 by Temple University researchers found out that helping children adjust to different sensations led to fewer autistic mannerisms and better behavior [8]. Our system supports figures, sounds, music, videos, tags and colorful presentations which promise to increase the interaction with smart environment and children's concentration during a learning task. Using sensor technologies, the body movement is measured and all the data are saved in a data base. One of the skills that some of the children with ASD should work to improve is the "short attention span for most lessons" [9]. Animation and multimedia presentation of information or data are used to increase the interest of the children to learn.

We will use our system in order to create a suitable environment for children during evaluation process of their abilities in math. We propose the use the HDT principles to identify students' weakness/ strengths and their creativity traits and then use the smart environment to support students in their learning by creating targeted math lessons for them based on the evaluation results.

\subsection{JXTA-Overlay}

P2P communication between children, caregivers and therapists can be build using JXTA-Overlay Platform. JXTAOverlay project is an effort to use JXTA technology for building an overlay on top of JXTA offering a set of basic primitives (functionalities) that are most commonly needed in JXTA-based applications. The proposed overlay comprises the following primitives: peer discovery, peer's resources discovery, resource allocation, task submission and execution, file/data sharing, discovery and transmission, instant communication, peer group functionalities (groups, rooms etc.), monitoring of peers, groups and tasks. The overlay is built on top of JXTA layer and provides a set of primitives that can be used by other applications, which on their hand, will be built on top of the overlay, with complete independence.

The most important part in a P2P system is the communication between peers. By using JXTA-Overlay, it is possible to overcome, firewalls, routers, NATs, and bridges in the private networks.

\subsection{SmartBox}

The implemented system the use of SmartBox has shown positive effects by increasing the learner's motivation and concentration during online learning. The research has shown that many students with autism have social interaction challenges that are very difficult to address outside real social situations. Interactive learning using computers can be 
used in order to provide low stress social practice [10], [14]. Computer use can provide students with a sense of control and consistency. In our previous work, we used our system in order to get and maintain the child's attention by obtaining the calm-alert state window in which our ability to function is maximized [11], [15]. In this state the child's nervous system is sufficiently aroused for peak attention and task performance which enhances a person's ability to register and orient to sensory information. The SmartBox device is integrated with our system as a useful tool for monitoring and controlling children activities. The size of the SmartBox is $35 \times 7 \times 12 \mathrm{~cm}$ (see Fig. 3). The SmartBox has the following sensors and functions:

- Body Sensor for detecting body and hand movement.

- Chair or Bed Vibrator Control for vibrating the chair or bed.

- Light Control for adjusting the room light.

- Smell Control for controlling the room smell.

- Sound Control to emit relaxing sounds.

- Remote Control Socket for controlling AC 100V socket (on-off control).

Figure 3: SmartBox functions.

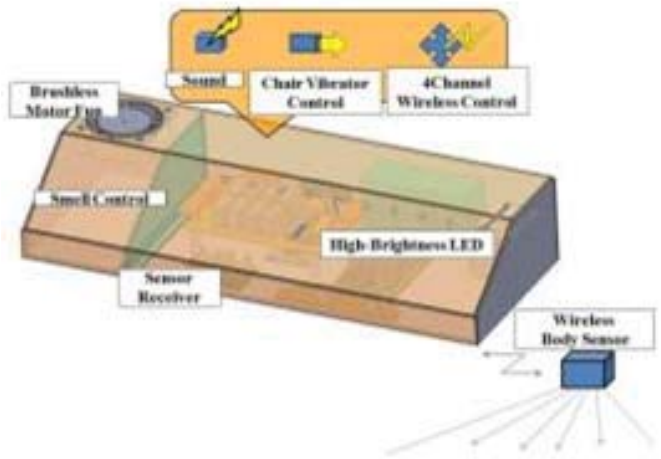

These functions can calm and relax children with ASD that are anxious or on a panic attack and increase concentration on tasks. ATs are often presumed to improve health and social care services for children with autism spectrum disorders. Researchers are taking in consideration that difficulties in sustaining attention on imposed tasks may be attributable partly to a developmental delay and partly to the motivational contingencies of a task rather than to a primary impairment in the ability to sustain attention [12].

In order to keep the child motivated in learning activities we used the features of SmartBox device such as the Chair Vibrator Control, Light Control, Smell Control, Sound Control.

- Chair or Bed Vibrator Control for vibrating the chair. Through sensory integration, physical vibrations of chair will relax and calm the child.

- Light Control for adjusting the room light. If the child is a visual learner, in order to capture child's attention we can use the computers screen to show colored images and use the light control for changing the room light.

- Smell Control for controlling the room smell. If the child likes certain smells/perfumes we can put the perfume of that smell to get the attention.

- Sound Control to emit relaxing sounds. If the child accepts auditory stimuli we can use this to get his attention and maintain focus in learning.

Tagging physical objects to find and analyze data about the object is one way the loT can be used in education. Using our proposed and implemented system (see Fig. 4), a child can learn new words through touching the physical objects that are in their vocabulary list. Each physical object would have a RFID tag placed on the item. When this tag is read by a RFID reader or scanned by an application running on a computer or mobile device it would prompt the device to open up a page of information or send a command for an action to happen.

RFID tags can be created and attached by the parents for each of the physical items in the vocabulary list. When the child places the RFID card on the RFID reader, it will say the word for the item in their native language. Touching the item will give to the child another sense to be engaged and may help them learn new words faster. 
Figure 4: Proposed smart environment

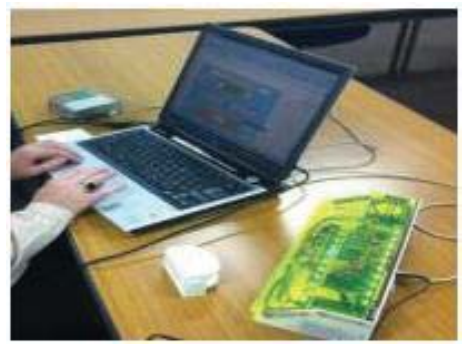

Every IC Tag card can be connected with Powerpoint slides (one IC Tag for one slide). All information is saved in an Access database. Every time that IC Tag card touch IC Tag reader (see Fig. 5), the slide connected with this card is displayed on computer screen (except computer screen also video projector can be used).

Figure 5: IC Tag card and IC Tag reader.

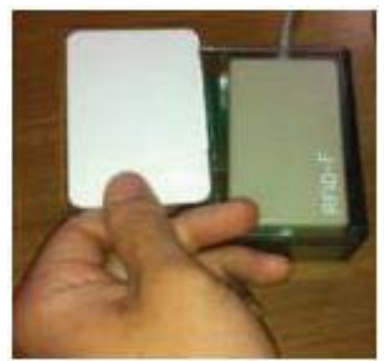

Our system can generate random presentation and flash presentation (the interval of flash presentation can be set manually). IC Tag cards can be shuffled as playing cards.

The experimental results showed the following:

- The smart environment can help children with autism stay focused during their learning and can maximize their ability to reach their peak attention.

- The child's task performance is enhanced and the child will be able to learn new language skills, social skills, appropriate behavior and academic skills.

- The child's concentration and the time amount of the study are improved by the usage of SmartBox.

\section{Proposal of Using Combination of Smart Environment and HDT Principle}

Many students have difficulties understanding math concepts and researchers and educators try to identify the main factors that affect their ability to learn in math subject. One of the methods used to diagnose student's weakness and strength in math is using the multi-step approach principles of HDT. The HDT is a pedagogy that investigates characteristics, gaps in content, and designing instruction to accommodate learner and the content to be learned [13]. The HDT involves the assessment of the performance level of a learner and understanding all of the factors that affect the performance level. The importance of this diagnoses is to select and develop the appropriate pedagogy that supports students' learning in math by taking under consideration important factors like generic influences on learning (social and emotional, cognitive, physical and sensory, psychomotor factors), socioeconomic status, as well as cultural, and family background.

We propose to use the SmartBox system and principles of HDT to identify the strengths and weakness in math of students with ASD and use the smart environment to support students learning in math. Teaching Mathematics to children with Autism can be very challenging when relying on traditional teaching methods and resources, which often encourage the use of 'mental strategies' as early as possible. However, with some creative thought and an emphasis on visual aids and personal interests, teaching Mathematics to children with Autism can be very successful and enjoyable for both the teacher and child [14]. 
Children with diagnosis of autism vary greatly in their strengths/weaknesses, likes/dislikes and general personality and approach to life. There is no one teaching approach that will be successful with all children who fall into this diagnostic category. However, there are a variety of conventional math programs and ideas out there that can be modified according to an individual child's profile of skills and interests, to help that child learn math concepts with meaning [15].

Using the proposed system to identify their abilities in math will help teachers develop math lessons modified to each student needs and use this system to support them during their learning. By using the SmartBox system we can get and maintain the child's attention by obtaining the calm-alert state window in which our ability to function is maximized. The reach of calm alert state window will allow for better evaluation due to the increase of child's ability to concentrate on a given task. Based on student's assessment the teacher can develop math lessons that target improvement of student's weakness in math and use the smart environment as a supportive environment to increase student's motivation during learning. By using the system the child's task performance is enhanced and the child will be able to learn new math skills. Most difficult aspects of math for ASD students are "language of math" (words describing mathematical concepts), word problems (accurately translating from language to mathematical problem), estimation, and prediction. Because many of children with ASD have language weakness, they will mostly have difficulties with word problems. We can use the proposed smart environment to translate the words into picture through the use of IC tags that present the information in pictures, symbols, visual concepts and later translate this information to equations to solve the math problem.

In the school system, math skills are traditionally taught through language. For a child with weak language skills, the verbal explanations of math concepts are difficult to understand. The use of the smart environment will support learning new math skills by using different pictures, symbols and visual concepts.

Solving word problem might be a difficult task for some students. Some of the reason that the word problems are difficult to be solve are that the students need to understand the problem and find out what is given and what is wanted. It will be easier for them to have the equation ready rather than trying to find out the equation first and find the solution. Knowing for fact that word problems are part of everyday life we can't avoid teaching children how to deal and solve these types of problems.

First, we should give students to solve easy word problems and check their ability to understand these problems. Second, we should teach those steps that they should take to be successful with these types of problems. Finally, we should check for understanding to make sure that they can use the needed tools to solve these problems. Dealing with word problems students will become more logically involved because of thinking that takes place. Our proposed smart environment supports the use of different tools to represent the information given and what is asked through charts and in this way it is easier to create the steps that are needed to solve a problem. In the future they will seek patterns to solve word problems and will use these patterns to deal with real life problems.

The proposal of the use of the HDT principles to support students with autism in their mathematics learning is based on the previous data received from the Annual Report, 2000 of the Drexel Diagnostic Mathematics and Science Learning. The data shows that the students that were taught using the HDT approach had improvement in their achievement in math subject [16].

The Heuristic Diagnostic Teaching (HDT) is an evaluation process used to identify student strengths, creativity and weaknesses, particularly in mathematics. The HDT process includes five principles which are used to evaluate students learning abilities in math; Identify Strengths and Weaknesses; Hypothesize Reasons for Strengths and Weaknesses; Formulate Behavioral Objectives; Develop Instruction; and Employ Ongoing Evaluation [17].

In order to identify the strength and weakness in students ability to learn in math subject we propose to use different tests like Piaget Conservation Tasks, Aiken Mathematics Attitude Assessment, Semantic Differentials, WISC-R type Coding Task, Feuerstein Logic Assessment and Feuerstein Drawing/Memory Assessment, math problems.

When we test students we should check for the disabilities that they have in process of learning. Knowing these problems we designate lessons that target these specific deficiencies. Building student profile knowing student's weaknesses help for a better understanding during communication teacher-student and student-content.

The HDT process involves the evaluation of the student's ability to learn in math and understanding all generic influences on students learning. By creating this picture about the student the teachers can develop learning strategies that target both the students' weakness and the generic influences on learning. Identification of learners' characteristics such as creative strengths are very important in this process because these characteristics can play an important role on student's ability to learn.

Mathematical creativity at classroom settings is defined as a) the process that results in novel and / or insightful solutions and b) the formulation of new questions and/or possibilities that allow an old problem to be regarded from a 
new point of view [18].

Creativity is a very powerful tool in process of learning because empowers student with self-esteem and gives them power to seek new ways of understanding the given data and finding a solution to given problems.

\section{A Case Study to Evaluate Students in Math}

\subsection{Assessments}

In the proposed case study, we will use as Assessment the Testing and Student Background.

The used Assessments are:

Piaget Conservation Tasks, Aiken Mathematics Attitude Assessment, Semantic Differentials, WISC-R Type Coding Task, Feuerstein Logic Assessment and Feuerstein Drawing/Memory Assessment, Two math problems from Math Forum.

In following, we will give some definitions.

\subsubsection{Piaget Conservation:}

Piaget's theory of conversation is the theory of conservation of identity. The purpose of the Piaget Conservation tasks is to confirm that the student is a conserver.

\subsubsection{Aiken Mathematics Attitude Assessment}

The assessment of attitude toward mathematics in students learning ability. The purpose of the Aiken's Mathematics Attitude Assessment is the affective assessment of student's feelings about mathematics.

Attitude scales were constructed to measure 2 aspects of the appreciation and use of mathematics. The 11-item $\mathrm{E}$ Scale is designed to assess the degree of enjoyment of mathematics and the 10-item V Scale is designed to measure recognition of the importance and relevance of mathematics.

The mathematics attitude scale, measures the general physiological dimension of "enjoyment of mathematics" [19], [20].

\subsubsection{Semantic Differentials}

Semantic differential is a technique for measuring meaning and relates to the functioning of representational process in language behavior. The Semantic differential is essentially a combination of controlled association and scaling procedures. The purpose of the Semantic Differential is the affective assessment of student's attitudes toward family, friends, school, and himself.

\subsubsection{WISC-R type Coding Task}

WISC-R = Wechsler Intelligence Scale for Children - Achievement Test - Revised

\subsubsection{Feuerstein Logic Assessment and Feuerstein Drawing/Memory Assessment}

The assessment of abilities and potential of a person that takes into account the background culture and historical changes [21].The purpose of the Feuerstein - Logic is to observe student for generic influences on learning, including ability in general to abstract and cope with complexity, problem-solving strategies and spatial relationships (left-right). The purpose of the Feuerstein - Drawing/Memory is to observe problem-solving strategies, attention to salient aspects (visual detail), and short-term memory.

5.1.6 Math problems used for testing based on students grade and age. 


\subsection{Testing Situation and Test Environment}

We will observe the student during different sessions. Interviewing the student in order to get to know better the student and to see what the student likes or dislikes, not only about math but other subjects too. Later, student can work on a test and observe the student during test taking. Then, the student can be observed during the time that he works on solving math problems.

During interviews with the parents, caregivers, teachers, student we should collect background information about the students regarding students' family members, siblings, and his attitude toward school in general and math subject, his mathematics ability and achievement. This will help us identify the generic influences on student's learning.

\section{Conclusions and Future Work}

In this paper, we proposed the use of smart environment and HDT principle in order to identify abilities in math for students diagnosed with ASD. We proposed the use of smart environment to better support the students during their learning by actively engaging the students during their learning and creating and maintaining their calm alert state.

Also, in order to identify the students' weakness and strengths in math we proposed different assessment tools.

In the future, we would like to implement our proposed framework and evaluate the assessment results to design math learning objectives and lessons for the students.

Also, in the future we would like to use the proposed system to improve the quality of life for children with ASD.

\section{References}

[1] American Psychiatric Association, "Diagnostic and Statistical Manual of Mental Disorders", 4th Edition. Washington, DC, 2000.

[2] K. Tucker, Teaching Mathematics to children with Autism, http://www.udg.edu/grupsrecerca/AutismeUdG/Recursoseducatius /Recursosperaprofessionals/Matematiquesiautisme/tabid/14306/language/en-S/Default.aspx

[3] Sheila Bell "Teaching Math with Meaning", http://www.autismandtheartofcommunication.com/files/Download/Teaching\%20Math \%20with\%20Meaning.pdf, January 2002

[4] Tomlinson \& Allan, "Leadership for Differentiating Schools \& Classrooms", ISBN-13: 978-0-87120-502-5, 2000.

[5] Home Networking, http://www.iec.org.

[6] A. Tapus, M. Matarich, B. Scassellati, "The Grand Challenges in Socially Assistive Robotics", Robotics \& Automation Magazine, IEEE, vol. 14, no. 1, pp. 35-42, 2007.

[7] Y. Huang, G. Li, "Descriptive models for Internet of Things", Proc. of Intelligent Control and Information Processing International Conference (ICICIP-2010), pp. 483-486, 2010.

[8] "Sensory Treatment Yields Promising Results for Children with Autism", http://news.temple.edu/news/sensory-treat ment-yieldspromising-results-children-autism.

[9] "Students with Autism and their Communication Issues: Strategies and Interventions"; http://www.authorstream. com/Presentation/duitdu-1833346-students-autism-communication-issues/.

[10] "Assistive Technology Supports for Individuals with Autism Spectrum Disorder", Wisconsin Assistive Technology Initiative, February, 2009, http://www.wati.org/ content/supports/free/pdf/ASDManual- 1.pdf.

[11] C. Murray-Slutsky, B. A. Paris, "Exploring the Spectrum of Autism and Pervasive Developmental Disorders", Therapy Skill Builders, 2000.

[12] H. B. Garretson, D. Fein, L. Waterhouse, "Sustained Attention in Children with Autism", Journal of Autism and Developmental Disorders, Vol. 20, Issue 1, pp 101-114, 1990.

[13] Reisman, F. K. (1978). A guide to the diagnostic teaching of arithmetic, Second Edition, Columbus: Charles E. Merrill Publishing.

[14] K. Tucker, "Teaching Mathematics to children with Autism", http://www.udg.edu/ grupsrecerca/AutismeUdG/Recursoseducatius /Recursosperaprofessionals/Matematiquesiautisme/tabid/14306/language/en-US/Default.aspx.

[15] S. Bell, "Teaching Math with Meaning",http://www.autismandtheartofcommunication.com/files/Download/Teaching\%20Math \%20with\%20Meaning.pdf, January 2002.

[16] L. A. Whitelaw, "An Evaluative Study of Teacher Creativity, use of the Heuristic Diagnostic Teaching Process and Student Mathematics Performance", Drexel University.

[17] F. K. Reisman, "A guide to the diagnostic teaching of arithmetic", Second Edition, Columbus: Charles E. Merrill Publishing, 1978.

[18] I. Pelczer, F. Gamboa Rodríguez, "Creativity assessment in school settings through problem posing tasks", Montana Mathematics Enthusiast [Serial online], January 2011, 8 (1/2) 383-398. Available from: Academic Search Premier, Ipswich, MA. Accessed November 22, 2013.

[19] Lewis R. Aiken, Jr., Attitudes toward Mathematics, Review of Educational Research Vol. 40, No. 4, Science and Mathematics Education, pp. 551-596, Published by: American Educational Research Association, 1970.

[20] L. R. Aiken, Jr, "Two Scales of Attitude toward Mathematics", Journal for Research in Mathematics Education, Vol. 5, No. 2 (Mar., 
1974), pp. 67-71 Vol. 5, No. 2 (Mar., 1974), pp. 67-71, Published by: National Council of Teachers of Mathematics; http://www.jstor.org/stable/748616.

[21] H. C. Haywood, D. Tzuriel, "Interactive Assessment", Springer, 1992. 